\title{
A mathematical framework simulating nerve fiber physiology
}

\author{
M. Z. Ul Haque ${ }^{1,2, *}$, Peng Du ${ }^{2}$, Leo K. Cheng ${ }^{2}$ \\ ${ }_{1}^{1}$ Department of Biomedical Engineering, Barrett Hodgson University, Karachi, Pakistan \\ ${ }^{2}$ Auckland Bioengineering Institute, University of Auckland, Auckland, New Zealand
}

\section{ARTICLE INFO}

\section{Article history:}

Received 15 June 2017

Received in revised form

20 August 2017

Accepted 29 August 2017

\section{Keywords:}

Action potential

Computational model

Diagnostic method

Electrophysiological nerve model

Lagrange basis function

Nerve conduction velocity

\begin{abstract}
A B S T R A C T
Nerves are an important part in human body which not only controls the movement and locomotion of the body but also but also contains sensory receptors from other parts of the body which provide continuous feedback to the brain and spinal cord. Various diagnostic methods are used to detect the specific damage response of the nerve but they do not identify the precise location of the nerve damage. A computational nerve model may help to identify the exact location of nerve damage. Therefore, in this study the organization of a one-dimensional (1D) synthetic single element structural and functional model which typify the anatomy and physiology of the nerve is proposed. The geometrical model was developed using 1D linear Lagrange basis function while the functional model was developed by applying external stimulus and solving the bidomain model. The unmyelinated and myelinated nerve electrophysiological models were used to generate and propagate the action potential in this 1D synthetic single element model (SSEM). The nerve conduction velocity (NCV) was also computed in this proposed model and found that the myelinated nerve model has a higher NCV in contrast to unmyelinated model. This model will provide a platform for the development of the complete anatomical and functional model of the nerves in the various location of the body and may be helpful for clinician and physiologists in the evaluation and diagnosis of the structural as well as functional consequences of diabetic neuropathy in its initial stages.
\end{abstract}

(C) 2017 The Authors. Published by IASE. This is an open access article under the CC BY-NC-ND license (http://creativecommons.org/licenses/by-nc-nd/4.0/).

The electrophysiology of nerves is of real importance here. A nerve which is a collected term for several hundred axons, plus associated blood vessels and connective tissue can either be classified as myelinated nerve or an unmyelinated nerve, depending upon the presence or absence of a multilayer lipid and protein layer around the nerve axon called the myelin sheath. This sheath provides electrical insulation and speeds up the nerve conduction velocity through the axon (Tortora and Derrickson, 2008). Myelinated nerve axons have increased diameters of up to $22 \mu \mathrm{m}$ resulting in enhanced conduction velocities of up to $130 \mathrm{~mm} \mathrm{~ms}^{-}$ 1 as compared to unmyelinated nerves having a small diameter of up to $1.3 \mu \mathrm{m}$ and slow conduction velocities of approximately $1.5 \mathrm{~mm} \mathrm{~ms}^{-1}$ (Tortora and Derrickson, 2008). This translates that the axon diameter and degree of myelination along with temperature governs the speed of nerve impulse propagation through a nerve fibre (Giuliodori and DiCarlo, 2004).

Diagnostic techniques for diabetic neuropathies include both invasive and non-invasive methods but lack the ability to locate the exact position of damage (Oh, 2007; Kennedy and Inglis, 2002; Oh, 1990; Lauria et al., 2005). Our potential solution to this 
problem is application of mathematics to neuronal structure and dynamics in a computer based medium; neuronal computational modelling (Dayan and Abbott, 2003). Such mathematical and computational models offer valuable tools for investigating nerve activity across multiple structural locations of the human body independent of the need for an actual patient.

Most neuronal mathematical models are electrophysiological in nature and there are separate models to analyze the electrical activity of unmyelinated and myelinated nerve fibres (Rattay and Aberham, 1993). Two of the most popular electrophysiological are the unmyelinated HodgkinHuxley (HH) and myelinated Chiu, Ritchie, Rogert, Stagg and Sweeney (CRRSS) nerve models respectively. The $\mathrm{HH}$ nerve model was the first comprehensive mathematical model of electrical excitability in the nerve fibre (Hodgkin and Huxley, 1952). HH defined the membrane potential from non-linear ordinary differential equations (ODEs) of a single neuron. In contrast, the CRRSS is suitable for simulating the myelinated peripheral sensory nerve fibre in rabbits (Chiu et al., 1979; Sweeney et al., 1987). In this model, experimentation was performed on the myelinated nerves for the measurement of quantitative membrane currents using the voltage clamp analysis (Chiu et al., 1979; Sweeney et al., 1987). They used a set of ODEs to determine the membrane potential. Formerly, the electrical stimulation in the retinal ganglion cell and human cochlear neuron was done by employing $\mathrm{HH}$ model respectively (Greenberg et al., 1999; Rattay et al., 2001). Whereas, the electrical stimulation in the sciatic nerve of the lower limb was achieved by implementing the CRRSS model (Kim et al., 2007).

Therefore, this research work describes the framework for the development of structural and functional model representing the anatomical and physiological behaviour of the nerves in the human body. This modelling framework presents a practical standard in which biophysical nerve activity can be included with the structural and functional aspects of the nerves in various location of the body. This may ultimately prove to be a major diagnostic tool for examining the pathological consequences of diabetic neuropathy at its initial stages.

\section{Methodology}

In this work, a 1D synthetic single element model (SSEM) is proposed and developed. This SSEM represents the anatomy and physiology of the nerve fibres and their details are given below:

\subsection{Geometrical nerve model}

In this study, a 1D geometrical SSEM with a length of $20 \mathrm{~mm}$, characterizing the structure of the nerve fibres was developed using a 1D linear Lagrange basis function in modelling software known as Continuum Mechanics, Image Analysis, Signal Processing and System Identification (CMISS).
The 1D linear Lagrange basis was selected due to the fact that nerve fibre act as a cylinder and model using 1D cable theory (Segev, 1998).

\subsection{Nerve electrophysiological model}

In this study, two nerve electrophysiological models namely unmyelinated $\mathrm{HH}$ and myelinated CRRSS models were used to simulate the action potential generation and propagation along the developed 1D SSEM respectively. These models were encoded using the CellML modelling benchmark (Hedley et al., 2001; Lloyd et al., 2004). Through these electrophysiological models, we were able to calculate the values of membrane potential, ionic currents, conductances, and their respective ordinary ODEs in CellML which resulted in the calculation of the action potential at a single grid point along the 1D SSEM. The details of these electrophysiological models are given as follows:

\subsubsection{HH model}

HH were the first physiologists who successfully experimented the behaviour of nerve excitation with squid giant axon using voltage clamp studies (Hodgkin and Huxley, 1952). The voltage gated channels of the cell membrane control the ion flow. The activation as well as inactivation gates of the channels permit the amount of influx and outflux of the ions responsible for the production of action potential. The equivalent electrical circuit of a $\mathrm{HH}$ model comprises of time dependent sodium and potassium channels, a time independent leakage channel, and a membrane capacitance, as shown in Fig. 1a.

- a: HH model equivalent electrical circuit representing the capacitor as the capacitance of the cell membrane, the voltage dependent sodium and potassium channels with arrows in their respective conductance symbol and voltage independent leakage channel with no arrow, the reverse potential of each channel i.e., $v_{N a 1}, v_{k 1}$ and $v_{L 1}$ represented by the round shaped battery symbol. The arrows for the ionic currents are indicated from inside to outside (Hodgkin and Huxley, 1952).

\subsubsection{CRRSS model}

The popular CRRSS model describes the mammalian myelinated nerve response. The nodes of Ranvier are modelled by voltage gated sodium channels and the voltage gated potassium channel are absent in CRRSS model (Chiu et al., 1979; Sweeney et al., 1987). The single cell myelinated nerve fibre model is represented in Fig. $1 \mathrm{~b}$.

- b: The equivalent cable electric cell model represents nodes of Ranvier in a CRRSS model. This model shows voltage dependent sodium (arrow) channel and a voltage independent 
leakage channel with no arrows. The reversal potential of each channel is designated by $\mathrm{v}_{\mathrm{Na} 2}$ and $\mathrm{v}_{\mathrm{L} 2}$, illustrated with a round shaped battery symbol. The arrows indicates the ionic currents

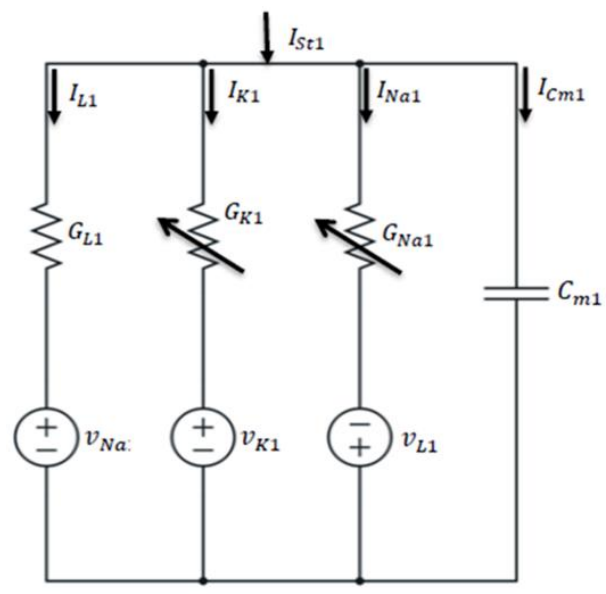

(a) flowing from inside to outside of the membrane (Chiu et al., 1979).

The parameters and constants used in the $\mathrm{HH}$ and CRRSS models for determining the action potential along the SSEM are given in Table 1.

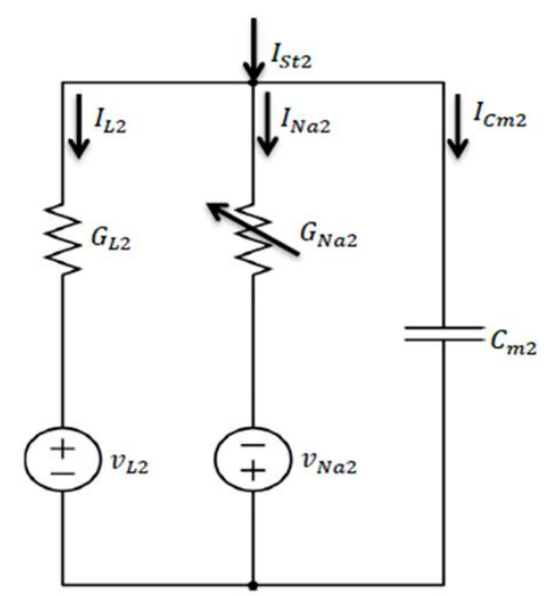

(b)

Fig. 1: Electrophysiological nerve electrical equivalent model

Table 1: Parameters and constants used in simulations of HH and CRRSS Models

\begin{tabular}{|c|c|c|}
\hline Parameters & HH Model & CRRSS Model \\
\hline & Values and units & Values and units \\
\hline Membrane capacitance $\left(\mathrm{v}_{\mathrm{m}}\right)$ & $0.01 \mu \mathrm{F} \mathrm{mm}^{-2}$ & $0.01 \mu \mathrm{F} \mathrm{mm} \mathrm{m}^{-2}$ \\
\hline Reverse potential $\left(\mathrm{v}_{\mathrm{r}}\right)$ & $-80 \mathrm{mV}$ & $-80 \mathrm{mV}$ \\
\hline Sodium reverse potential $\left(\mathrm{v}_{\mathrm{Na}}\right)$ & $115 \mathrm{mV}$ & $115.64 \mathrm{mV}$ \\
\hline Potassium reverse potential $\left(\mathrm{v}_{\mathrm{k}}\right)$ & $-12 \mathrm{mV}$ & N.A. \\
\hline Leakage reverse potential $\left(\mathrm{v}_{\mathrm{L}}\right)$ & $10.613 \mathrm{mV}$ & $-0.01 \mathrm{mV}$ \\
\hline Sodium maximum conductance $\left(\mathrm{v}_{\text {Namax }}\right)$ & $1.2 \mathrm{mS} \mathrm{mm}-2$ & $14.45 \mathrm{mS} \mathrm{mm}^{-2}$ \\
\hline Potassium maximum conductance $\left(\mathrm{v}_{\mathrm{kmax}}\right)$ & $0.36 \mathrm{mS} \mathrm{mm}^{-2}$ & N.A. \\
\hline Leakage maximum conductance $\left(\mathrm{v}_{\mathrm{Lmax}}\right)$ & $0.03 \mathrm{mS} \mathrm{mm}^{-2}$ & $1.28 \mathrm{mS} \mathrm{mm}^{-2}$ \\
\hline Nodal gap length (l) & N.A. & $1.3 \mathrm{~mm}$ \\
\hline Diameter of the fibre (d) & N.A. & $7 \mu \mathrm{m}$ \\
\hline
\end{tabular}

\subsection{Functional nerve model}

In order to translate the structural model into a functional model, a flow chart representing the various stages involved for action potential propagation (APP) along a SSEM is shown in Fig. 2. These stages are used for APP as well as NCV measurements along the 1D SSEM, characterizing the physiology of the nerve fibre. The geometrical 1D SSEM opted as an input for APP along the diverse parts of the elements. Grid points were allotted in the same vicinity as in the local coordinate space for this formed geometrical SSEM. The initial calculated values of the membrane potential, ionic currents and their respective ODEs obtained from the CellML based electrophysiological nerve models were passed to the equally space single grid points of this 1D SSEM. In finite element modeling (FEM), bidomain model (Tung, 1978) was selected to characterize the APP along the single grid point of the designed 1D SSEM by applying extracellular stimulus as it was formerly used to model neural tissue electrical activity (Kim et al., 2007). External stimulation was applied at time $\mathrm{t}=0$ and the membrane voltage value returned to the CellML based electrophysiological nerve model which computed updated values of membrane potential, ionic currents and their particular ODEs and this parameters value were once more reassigned to the bidomain model for computing newer values of the action potential. The LSODA numerical integration technique was implemented in order to solve the differential equation implicitly of the electrophysiological nerve models for ionic current (Hindmarsh, 1983) and compute the action potential of each grid point in the element iteratively to a final desired time interval. The final APP output obtained from the bidomain model was then exported to the front end of CMISS namely continuum mechanics graphical user interface (CMGUI) and to UNEMAP. CMGUI is used for the 3D visualization of APP along the 1D nerve geometrical model and APP traces at different location (grid points) were displayed in UNEMAP.

\section{Results and discussions}

The 1D SSEM illustrating the structure of the nerve fibre is shown in Fig. 3. This 1D element has a length of $20 \mathrm{~mm}$ and divided into regular, evenly space grid points.

For functional model development, the aforementioned methodology was implemented in Fig. 3. There were 70 grid points in this 1D SSEM 
that gave a spatial resolution of $0.29 \mathrm{~mm}$. Here, an assumption was done that the diameter remained steady along the entire 1D SSEM. An extracellular stimulus current with a magnitude of $40000 \mu \mathrm{A} \mathrm{mm}^{-1}$ and duration of $0.5 \mathrm{~ms}$ was injected into the left end of the nerve fibre model using bidomain model (1D element) as shown in Fig. 4.

This magnitude of extracellular stimulus was strong enough to depolarize the nerve fibre and cause the APP along the representative 1D element nerve model that used both the unmyelinated $\mathrm{HH}$ and myelinated CRRSS models. Fig. 4 illustrates that the membrane voltage is at resting potential at $0 \mathrm{~ms}$ (blue sphere) with no extracellular stimulus. The membrane potential then starts propagating from 0 ms onwards. This subsequent APP is shown in Fig. 4. As a whole, Fig. 4 represents 1D SSEM setup using linear Lagrange basis function representing the physiology of the nerve fibre with regular, evenly spaced $0.29 \mathrm{~mm}$ grid point space.

This is represented at $0 \mathrm{~ms}$, which is the resting state of the fibre. Action potential was propagated along a 1D SSEM from the leftmost grid point to the right end of the nerve fibres using $\mathrm{HH}$ model at various time steps i.e., $0.1 \mathrm{~ms}, 1 \mathrm{~ms}$, and $3 \mathrm{~ms}$.

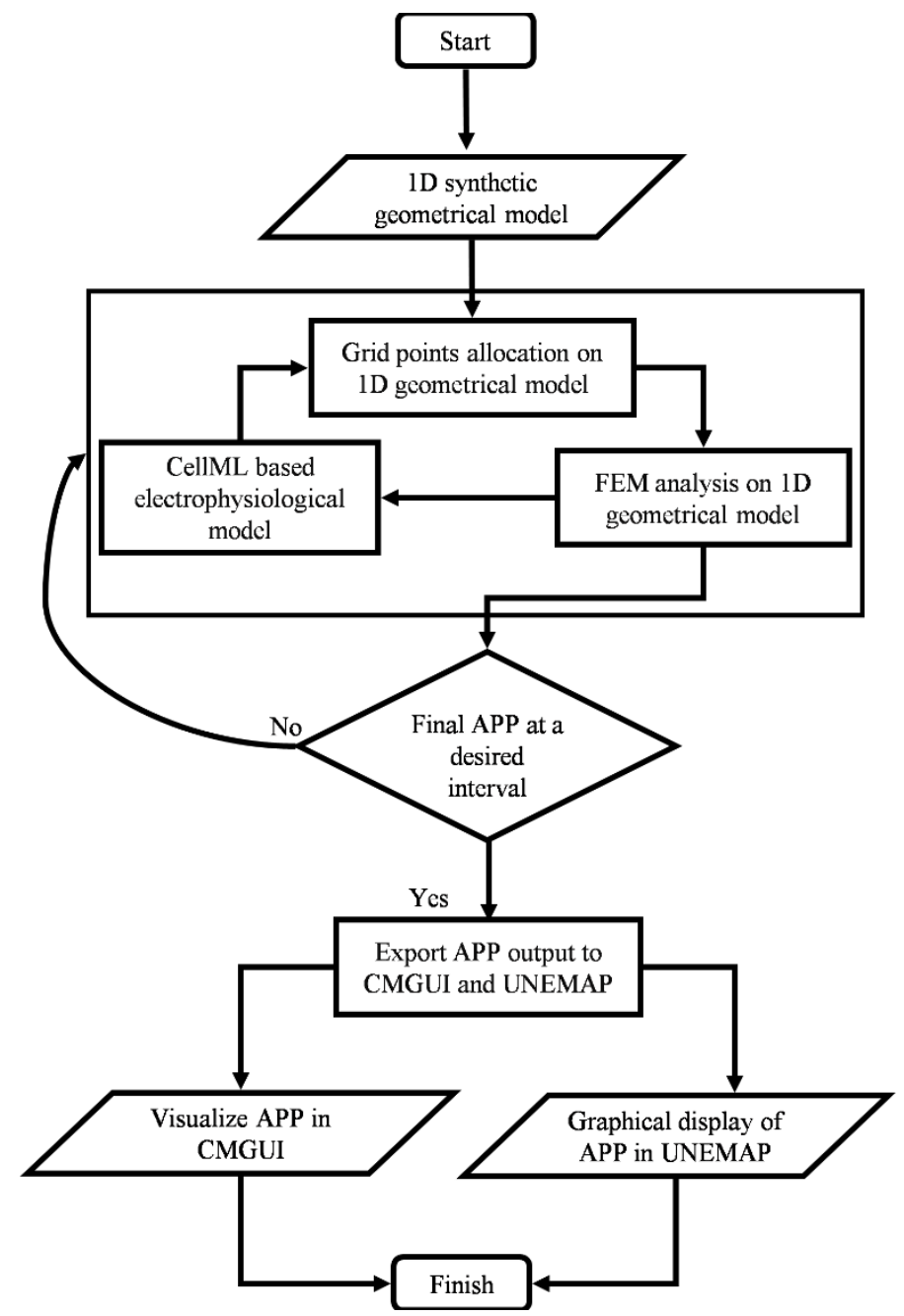

Fig. 2: Flow chart representing the functional model development for APP along the 1D SSEM

Fig. 3: 1D single element model with regular space grid points

The NCV was used to characterize the action potential movement along the entire length of the SSEM of the nerve fibre and to determine the convergence of the bidomain $1 \mathrm{D}$ SSEM. It is calculated by (Eq. 1),

$N C V=\frac{\sum D}{t_{f}-t_{s}}$

where, D indicates the sum of the distances of individual grid points along the 1D SSEM of the nerve fibre, $t_{f}$ represents the final time at which the maximum amplitude of the action potential reached the other boundary end of the SSEM of the nerve fibre and $t_{s}$ is the start time at which the extracellular stimulus current was applied to activate the bidomain nerve fibre.

The NCV at the various locations in the developed 1D SSEM was then determined using Eq. 1 using HH and CRRSS electrophysiological nerve models and is presented in Table 2 . From Table 2, it can be easily determined that the NCV in the developed 1D SSEM was higher using myelinated CRRSS nerve model in contrast to unmyelinated $\mathrm{HH}$ nerve model over the same location i.e., distance of this 1D SSEM. 


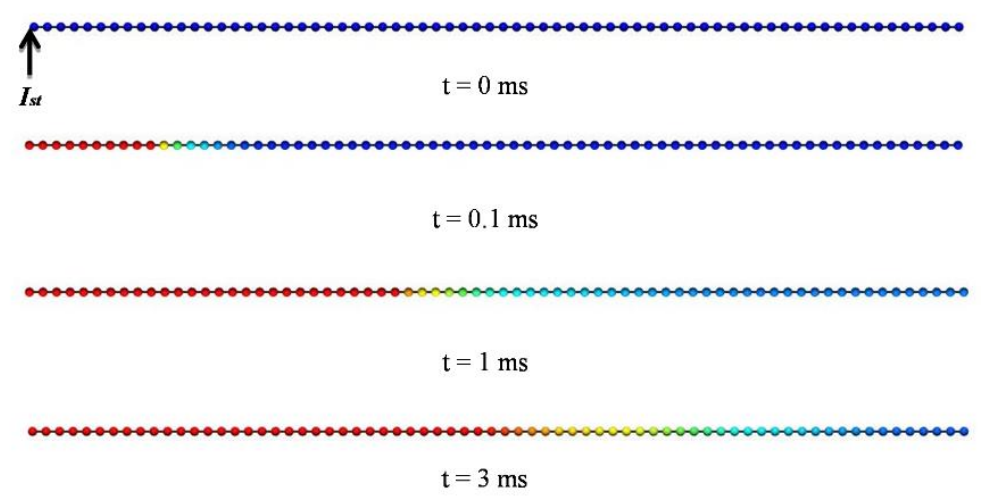

Fig. 4: 1D SSEM setup using linear Lagrange basis

Table 2: NCV measurements at different locations in the 1D SSEM using HH and CRRSS model

\begin{tabular}{ccccc}
\hline \multirow{2}{*}{ Distance $(\mathrm{mm})$} & \multicolumn{2}{c}{ HH Model } & \multicolumn{2}{c}{ CRRSS Model } \\
\cline { 2 - 5 } & Time $(\mathrm{ms})$ & $\mathrm{NCV}\left(\mathrm{mm} \mathrm{ms}^{-1}\right)$ & Time $(\mathrm{ms})$ & $\mathrm{NCV}\left(\mathrm{mm} \mathrm{ms}^{-1}\right)$ \\
\hline 4.3 & 1 & 4.3 & 0.2 & 21.5 \\
8.6 & 1.9 & 4.5 & 0.36 & 23.9 \\
12.9 & 2.8 & 4.6 & 0.5 & 25.8 \\
\hline
\end{tabular}

Furthermore, a simulation research was executed to obtain numerical convergence in this $20 \mathrm{~mm}$ length 1D SSEM by varying the spatial resolution of the grid points. So, the grid points varied between 20 points $(\Delta x=1 \mathrm{~mm})$ to 100 points $(\Delta x=0.2 \mathrm{~mm})$. The NCV was then determined using Eq. 1 based on this range of spatial resolution by applying the simulations iteratively with a nerve fibre diameter of $5 \mu \mathrm{m}$ and the intracellular and extracellular conductivities of $50 \mathrm{mS} \mathrm{mm}^{-1}$ respectively. The effect of spatial resolutions on NCV from both the unmyelinated $\mathrm{HH}$ model and the myelinated CRRSS model is shown in Fig. 5. It can be concluded from
Fig. 5 that the NCV declined as the number of spatial resolutions (grid point per $\mathrm{mm}$ ) increased. It stopped declining as soon as the spatial resolution convergence of $0.29 \mathrm{~mm}$ was reached for both the $\mathrm{HH}$ and the CRRSS models respectively. In addition, the convergence of NCV achieved from the CRRSS model was higher than the NCV obtained from the HH model. This might be due to the certainty that it has a myelin sheath and also contains nodes of Ranvier (Giuliodori and DiCarlo, 2004). The converged NCV was $25.6 \mathrm{~mm} \mathrm{~ms}^{-1}$ and $7.1 \mathrm{~mm} \mathrm{~ms}^{-1}$ for the CRRSS and the $\mathrm{HH}$ models respectively.

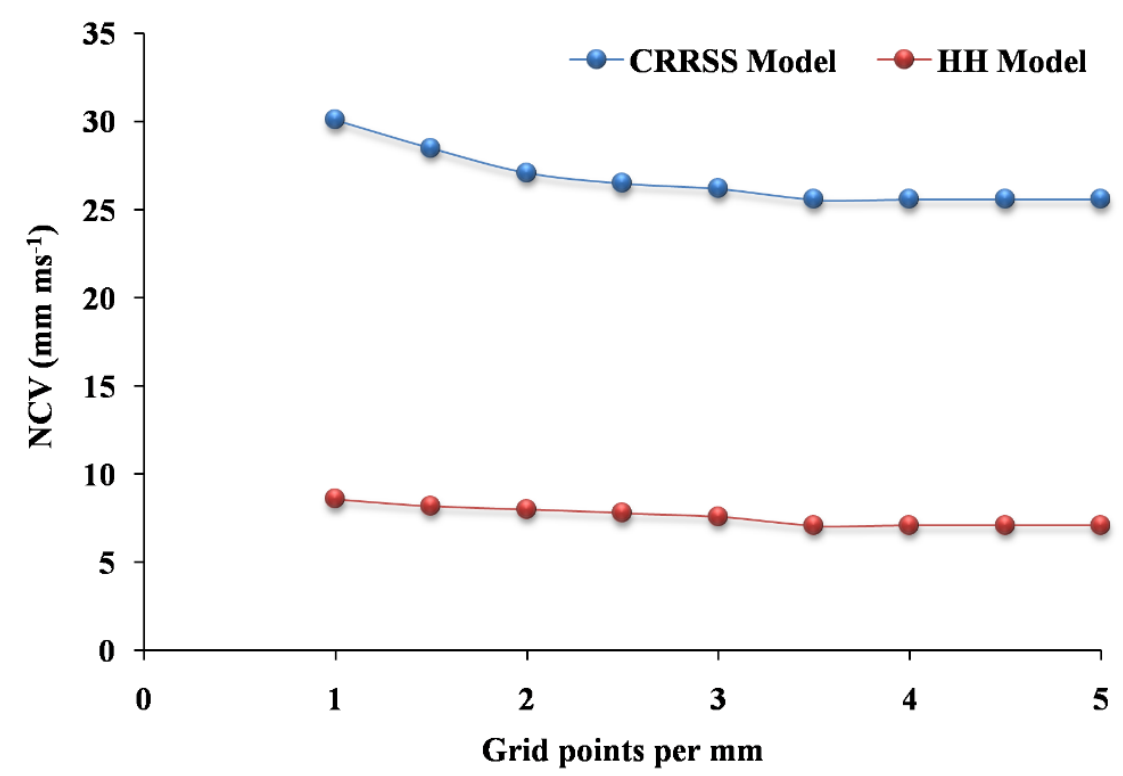

Fig. 5: Convergence test for the spatial resolution using the bidomain model to examine the NCV in a 1D SSEM using the CRRSS and the HH model. With the increase in grid points per $\mathrm{mm}$, the NCV was decreased. The minimum converged spatial resolution was $\sim 3.5$ grid point per mm or grid point spacing of $\sim 0.29 \mathrm{~mm}$

\section{Conclusion}

A framework for the development of structural and functional models, which embodies the anatomy and physiology of the single nerve fibre, has been described. This framework presents a podium for the development of computational nerve models of the nerve networks in different regions of the human 
body. These models can be used to examine normal and abnormal neuronal function. This can eventually prove to be a useful diagnostic tool for investigating the pathological outcomes of diabetic neuropathy such as diabetic ulcers and other related conditions. In the prospective works, an electromechanical coupling between loading and sensory nerve feedback can be made in healthy as well as diabetic neuropathy patients.

\section{Acknowledgement}

The authors acknowledge the financial support provided by New Economy Research Fund of New Zealand.

\section{References}

Chiu SY, Ritchie JM, Rogart RB, and Stagg D (1979). A quantitative description of membrane currents in rabbit myelinated nerve. The Journal of Physiology, 292(1): 149-166.

Dayan P and Abbott LF (2003). Theoretical neuroscience: Computational and mathematical modeling of neural systems. Journal of Cognitive Neuroscience, 15(1): 154-155.

Giuliodori MJ and DiCarlo SE (2004). Myelinated vs. unmyelinated nerve conduction: A novel way of understanding the mechanisms. Advances in Physiology Education, 28(2): 80-81.

Greenberg RJ, Velte TJ, Humayun MS, Scarlatis GN, and De Juan E (1999). A computational model of electrical stimulation of the retinal ganglion cell. IEEE Transactions on Biomedical Engineering, 46(5): 505-514.

Hedley WJ, Nelson MR, Bellivant DP, and Nielsen PF (2001). A short introduction to CellML. Philosophical Transactions of the Royal Society of London A: Mathematical, Physical and Engineering Sciences, 359(1783): 1073-1089.

Hindmarsh AC (1983). ODEPACK, a systematized collection of ODE solvers. In: Stepleman RS (Ed.), IMACS transactions on scientific computation, 1: 55-64. North-Holland, Amsterdam, Netherlands.

Hodgkin AL and Huxley AF (1952). A quantitative description of membrane current and its application to conduction and excitation in nerve. The Journal of Physiology, 117(4): 500544 .
Kennedy PM and Inglis JT (2002). Distribution and behaviour of glabrous cutaneous receptors in the human foot sole. The Journal of Physiology, 538(3): 995-1002.

Kim JH, Davidson JB, Röhrle O, Soboleva TK, and Pullan AJ (2007). Anatomically based lower limb nerve model for electrical stimulation. Biomedical Engineering Online, 6(1): 48-48.

Lauria G, Cornblath DR, Johansson O, McArthur JC, Mellgren SI, Nolano M, and Sommer C (2005). EFNS guidelines on the use of skin biopsy in the diagnosis of peripheral neuropathy. European Journal of Neurology, 12(10): 747-758.

Lloyd CM, Halstead MD, and Nielsen PF (2004). CellML: Its future, present and past. Progress in Biophysics and Molecular Biology, 85(2): 433-450.

McKusick VA (2007). Mendelian Inheritance in Man and its online version, OMIM. The American Journal of Human Genetics, 80(4): 588-604.

Oh SJ (1990). Diagnostic usefulness and limitations of the sural nerve biopsy. Yonsei Medical Journal, 31(1): 1-26.

Oh SJ (2007). Neuropathies of the foot. Clinical Neurophysiology, 118(5): 954-980.

Rattay F and Aberham M (1993). Modeling axon membranes for functional electrical stimulation. IEEE Transactions on Biomedical Engineering, 40(12): 1201-1209.

Rattay F, Lutter P, and Felix H (2001). A model of the electrically excited human cochlear neuron: I. contribution of neural substructures to the generation and propagation of spikes. Hearing Research, 153(1): 43-63.

Ristow M (2004). Neurodegenerative disorders associated with diabetes mellitus. Journal of Molecular Medicine, 82(8): 510529.

Segev I (1998). Cable and compartmental models of dendritic trees. In: The Book of GENESIS. Springer, New York. https://doi.org/10.1007/978-1-4612-1634-6_5

Sweeney JD, Mortimer JT, and Durand D (1987). Modeling of mammalian myelinated nerve for functional neuromuscular stimulation. In the $9^{\text {th }}$ Annual Conference on Engineering in Medicine and Biology Society. IEEE, 1577-1578.

Tortora GJ and Derrickson BH (2008). Principles of anatomy and physiology. John Wiley and Sons, Hoboken, New Jersey, USA.

Tung L (1978). A bi-domain model for describing ischemic myocardial dc potentials. Ph.D. Dissertation, Massachusetts Institute of Technology, Massachusetts, USA.

Vinik A, Ullal J, Parson HK, and Casellini CM (2006). Diabetic neuropathies: clinical manifestations and current treatment options. Nature Reviews: Endocrinology, 2(5): 269-281. 